\title{
INJÚRIAS DENTÁRIAS EM DENTES DECÍDUOS: ESTUDO LONGITUDINAL
}

\section{Marina de Lourdes Calvo Fracasso}

Docente Associada e Coordenadora do Programa de Residência em Odontopediatria da Universidade Estadual de Maringá (UEM); Docente do Programa de Pós-graduação em Odontologia Integrada Mestrado na Universidade Estadual de Maringá (UEM), Maringá (PR), Brasil.

E-mail: mafracasso@gmail.com

\section{Gabriela Cristina Santin}

Doutora em Odontologia pela USP/FORP; Docente Adjunta na Universidade Estadual de Maringá (UEM), Maringá (PR), Brasil.

\section{Gabriela Machado de Oliveira Terra}

Residente na modalidade especialização em Odontopediatria pela Universidade Estadual de Maringá (UEM), Maringá (PR), Brasil.

\section{Graziela Martioli}

Residente na modalidade especialização em Odontopediatria pela Universidade Estadual de Maringá (UEM), Maringá (PR), Brasil.

\section{Maria Gisette Arias Provenzano}

Doutora em Odontologia pela Universidade Norte do Paraná (UNOPAR); Docente Adjunta Universidade Estadual de Maringá (UEM), Maringá (PR), Brasil.

\section{Andressa Camilo}

Discente no curso de Odontologia Universidade Estadual de Maringá (UEM), Maringá (PR), Brasil.

\section{Sandra Mara Maciel}

Doutora em Saúde Pública pela USP; Docente do Programa de Pós-graduação em Odontologia Integrada na Universidade Estadual de Maringá (UEM), Maringá (PR), Brasil.
RESUMO: O objetivo deste estudo foi avaliar 139 crianças, com histórico de traumatismo em dentes decíduos, verificando as sequelas e a associação com gênero e idade. $33,8 \%$ da amostra apresentavam entre 24 e 35 meses no momento do trauma e $61,2 \%$ do gênero masculino. Quanto ao tipo de injúria dental e tecido de suporte, as mais prevalentes foram a fratura de esmalte $(28,1 \%)$ e a luxação lateral (17,3\%). Na dentição decídua, diagnosticou-se descoloração da coroa $(18 \%)$, reabsorção radicular inflamatória $(7,9 \%)$ e lesão periapical (6,5\%). $13,7 \%$ dos dentes permanentes estavam irrompidos, sendo 2,9\% com hipoplasia de esmalte; radiograficamente, 2,2\% apresentou atraso na erupção e $2,2 \%$ a posição anormal do dente. O Teste Quiquadrado mostrou associação da idade no momento do trauma com injúria dentária $(p=0,02)$; tipo de injúria dentária $(p=0,01)$ e sequelas clínicas nos dentes decíduos $(p=0,02)$. Conclui-se que é elevada a prevalência de sequelas diagnosticadas no acompanhamento, demonstrando a importância da preservação.

PALAVRAS-CHAVE: Traumatismo dentário; Dentição; Dentição permanente.

\section{DENTAL INJURIES IN DECIDUOUS TEETH: A LONGITUDINAL STUDY}

\begin{abstract}
Current study analyzes 139 children with a history of traumatism in deciduous teeth through sequelae and the association between gender and age. Further, $33.8 \%$ of the sample showed that the trauma started between 24 and 35 months, with $61.2 \%$ involving males. Regarding to type of dental injury and tissue, enamel fracture $(28,1 \%)$ and lateral dislocation $(17,3 \%)$ were the most prevalent. De-coloration of the crown (18\%), inflammatory radicular reabsorption $(7,9 \%)$ and peri-apical lesion $(6,5 \%)$ were diagnosed in deciduous dentition. Moreover, $13,7 \%$ of permanent teeth had emerged, with 2,9\% featuring enamel hypoplasia, whereas $2,2 \%$ showed a radiographic delay in eruption and $2,2 \%$ revealed abnormal position of teeth. Chi-square test demonstrated a link at the moment of trauma with dental injury $(p=0,02)$; type of dental injury $(p=0,01)$ and clinical sequelae in deciduous teeth $(p=0,02)$. Results show high prevalence of sequelae diagnosed in the follow-up and thus the importance of preservation.
\end{abstract}

KEY WORDS: Tooth Injuries; Dentition ; Dentition; Permanent. 


\section{INTRODUÇão}

Os traumatismos dentários ocorrem com elevada frequência na infância devido ao início do desenvolvimento da marcha e as quedas decorrentes desse processo, sendo essa a segunda razão mais comum das visitas aos Odontopediatras. (CARVALHO; JACOMO; CAMPOS, 2010; GOVINDARAJAN et al., 2012; BÜCHER et al.,2013; COSTA et al., 2014; MENDONZA-MENDONZA et al., 2015; BERTI et al., 2015). Dados estatísticos demonstram que um terço das crianças pré-escolares já sofreram traumatismo dentário envolvendo a dentição decídua (ELKARMI et al., 2015), e quase um quarto de todas as crianças que estão na escola já sofreram algum tipo de acidente envolvendo a dentição permanente (TOPRAK et al., 2014). Para Zhanget al. (2014) e Atabek et al. (2014), a injúria de um dente decíduo pode ter um efeito psicológico bastante negativo para as crianças e os pais, devido ao comprometimento estético, com reflexos sobre a qualidade de vida, além de ocasionar alterações funcionais no arco dentário (HOLAN; NEEDLEMAN, 2014; VIEGAS et al., 2014), exigindo do profissional conhecimento técnico científico, e em alguns casos o acompanhamento por uma equipe multidisciplinar (MALMREN et al., 2012).

Após a ocorrência de uma injúria nos dentes decíduos, é possível o surgimento de complicações ou sequelas tanto para os dentes decíduos como para os sucessores permanentes, sendo muitas vezes imperceptíveis inicialmente, todavia com manifestações mais complexas com o decorrer do tempo (ASSUNÇÃO et al., 2009; JÁCOMO; CAMPOS, 2009; CARVALHO; JACOMO; CAMPOS, 2010; OZDEMIR; AKIN; EDEN, 2011; QASSEM et al., 2014). Destaca-se a descoloração da coroa, necrose pulpar, hiperemia, calcificação pulpar, reabsorção inflamatória e anquilose são as sequelas mais prevalentes para os traumas de dentes decíduos (CARDOSO; ROCHA, 2010; CARVALHO; JACOMO; CAMPOS, 2010). Alguns traumas ocorridos na dentição decídua podem atingir o germe do dente permanente em desenvolvimento, visto que este é separado da região periapical do dente decíduo por uma barreira de tecido duro cuja espessura é inferior a 3mm de espessura (ALTUN et al., 2009; CARVALHO; JACOMO; CAMPOS, 2010; GÜNGÖR; PÜŞMAN; UYSAL,
2011; AMORIM; ESTRELA; COSTA, 2011). Segundo relatos científicos o que pode ser associadas a diversos fatores, como a idade da criança no momento do trauma, o grau de reabsorção radicular do dente decíduo lesionado, o tipo e a extensão da lesão traumática, e o estágio de desenvolvimento do germe do dente permanente (ALTUN et al., 2009; GOMES et al., 2010; CARVALHO; JACOMO; CAMPOS, 2010; GÜNGÖR; PÜŞMAN; UYSAL, 2011; AMORIM; ESTRELA; COSTA, 2011; QASSEM et al., 2014). As sequelas no sucessor permanente podem provocar desde uma descoloração ou uma hipoplasia de esmalte, até a dilaceração da coroa ou raiz, odontoma, sequestro do germe do permanente, parcial ou total interrupção da formação da raiz e distúrbios de erupção (ASSUNÇÃO et al., 2009; CARVALHO; JACOMO; CAMPOS, 2010; GOMES et al., 2010; GÜNGÖR; PÜŞMAN; UYSAL, 2011; AMORIM; ESTRELA; COSTA, 2011; SKAARE; AAS; WANG, 2013).

Sendoassim, tantoos profissionais deodontologia quanto os pais devem se atentar sobre a importância do tratamento após uma lesão traumática, e principalmente sobre a importância do acompanhamento clínico e radiográfico a médio e longo prazo do dente decíduo lesionado, bem como do seu sucessor permanente, a fim de que seja realizado o diagnóstico de possíveis sequelas em tempo oportuno para uma reabilitação adequada e melhor prognóstico. Para Arikan, Sari, Sonmez (2010); Atabeket al. (2014) e Elkarmi et al. (2015) é altamente recomendável planejar campanhas voltada para os pais, crianças e, profissionais da saúde com ênfase na importância da prevenção do trauma dentário e como tratá-lo prontamente.

$\mathrm{Na}$ literatura embora muitas pesquisas relatem experiências cientificas em vários países e estados brasileiros (KRAMER et al., 2003; ASSUNÇÃO et al., 2009; JESUS et al., 2010; COUTINHO; CAJAZEIRA, 2011; BÜCHER et al., 2013; VIEGAS et al., 2015; BERTI et al.,2015; ELKARMI et al., 2015), não existem estudos conduzidos para determinar dados sobre traumatismos dentários na dentição decídua na região norte do Estado do Paraná (PR), Brasil. Diante do exposto, o objetivo do presente trabalho foi determinar a prevalência, conduta clínica, bem como avaliação de sequelas nos dentes decíduos envolvidos e sucessores permanentes, em crianças atendidas na Clínica Odontológica da 
Universidade Estadual de Maringá-PR, no período entre 2011 a 2015. Foram também avaliadas a associação entre as variáveis relacionadas ao trauma com gênero e idade das crianças no momento do traumatismo.

\section{METODOLOGIA}

O presente trabalho foi submetido ao Comitê Permanente de Ética em Pesquisa Envolvendo Seres Humanos da Universidade Estadual de Maringá, Parecer Número 1.144.717 (CAAE 43711315.0.0000.0104).

O estudo foi realizado por meio de uma análise retrospectiva, a partir de prontuários de pacientes infantis atendidos na Clínica odontológica do Curso de Odontologia da Universidade Estadual de Maringá - PR, no período correspondente aos anos de 2011 a 2015. Foram selecionadas 139 crianças, que continham no seu prontuário histórico de trauma em dente decíduo e tratamento emergencial realizado na Clínica Odontológica da Universidade Estadual de Maringá PR. Os responsáveis pelas crianças foram contactados por telefone sendo reforçado a estes a importância da proservação dos dentes com histórico anterior de trauma. Em seguida foi agendada consulta de reavaliação do paciente em questão.

Todas as informações necessárias ao estudo, referentes aos dados da criança no momento do traumatismo como gênero, idade, causa do trauma, dentes envolvidos, tipo da injúria traumática, tempo decorrido para o primeiro atendimento e injúrias observadas já no primeiro atendimento foram coletadas do prontuário clínico de cada paciente. As injúrias traumáticas dos dentes decíduos e permanentes seguiu a classificação preconizada por Andreasen, Andreasen e Andersson, (2007).

Um único cirurgião dentista, devidamente calibrado, por meio de exames clínico e radiográfico, realizou a avaliação bucal de todas as crianças. Os atendimentos clínicos ocorreram na clínica odontológica, após consentimento do responsável pelo menor de idade com assinatura do Termo de Consentimento Livre e Esclarecido para Menores.

Em cada paciente, previamente ao exame foi realizada a profilaxia dentária e o exame clínico detalhado dos dentes envolvidos no trauma, por meio da palpação e percussão, além de tomadas radiográficas periapicais ou oclusais, com película radiográfica número 02. Após análise, os dados foram anotados em uma ficha específica para este fim. As sequelas clínicas e radiográficas dos dentes decíduos foram analisadas de acordo com o proposto por Andreasen, Andreasen e Andersson, (2007) considerando os seguintes aspectos: Sinais clínicos: ausência de sinais clínicos; descoloração da coroa, abcesso e fístula; Sinais Radiográficos: ausência de alteração radiográfica, lesão periapical, reabsorção inflamatória, calcificação pulpar, anquilose dentária.

Para análise das sequelas clínicas e radiográficas nos dentes permanentes foram considerados os seguintes sinais clínicos: descoloração da coroa, com esmalte hipoplásico, dilaceração da coroa do dente; Sinais radiográficos: dilaceração da raiz do dente, odontoma, duplicação da raiz, desenvolvimento incompleto da raiz, má posição do dente permanente.

Nos casos onde ficaram diagnosticadas alterações patológicas em dentes decíduos ou permanentes, os pacientes foram encaminhados para a disciplina de Odontopediatria onde receberam tratamento clínico adequado.

Os dados quantitativos foram analisados utilizando o programa Statistical Package for the Social Scienses (SPSS for Windows, versão 15.0, SPSS ${ }^{\circledR}$ Inc, Chicago,III), obtendo-se a frequência relativa (\%) das variáveis estudadas. Buscando determinar se havia associação entre as variáveis relacionadas ao trauma, os dados foram analisados segundo o gênero da criança, e a idade no momento do trauma, utilizando-se o Teste QuiQuadrado $(p<0,05)$.

\section{RESULTADOS}

Foram avaliados 139 pacientes com histórico de traumatismo dentário em dentes decíduos, totalizando 218 dentes. Houve maior prevalência entre crianças do gênero masculino $(61,2 \%)$ em relação ao feminino $(38,8 \%)$.

A idade das crianças no momento do traumatismo dentário variou de 1 a 6 anos, com média equivalente a 
2,58 anos de idade, sendo a idade mais prevalente entre 24 - 35 meses $(33,8 \%)$. Outro destaque importante foi que $75 \%$ crianças avaliadas sofreram trauma até 48 meses de idade.

O fator etiológico do traumatismo de maior prevalência foram as quedas em $74,8 \%$, atentando-se que para 7,2\% destas crianças já havia relatos de história prévia de traumatismo dentário.

Os incisivos superiores são envolvidos com frequência nos acidentes e somente 4 incisivos inferiores foram envolvidos em traumatismo. Em geral mais de um elemento dentário é atingido $(51,1 \%)$ no trauma.
A Figura 1 ilustra a prevalência das lesões traumáticas, envolvendo os dentes decíduos, no momento em que o trauma ocorreu. Quanto ao tipo de injúria ao dente, 52 (37,4\%) tiveram comprometimento da coroa dentária, $28,1 \%$ com fratura de esmalte, seguido da fratura de esmalte e dentina com exposição pulpar (5,0\%). Já para as injúrias aos tecidos de suporte, 90 crianças $(64,7 \%)$ apresentaram alteração, sendo mais diagnosticadas a luxação lateral 17,3\%, seguido da subluxação $12,2 \%$.

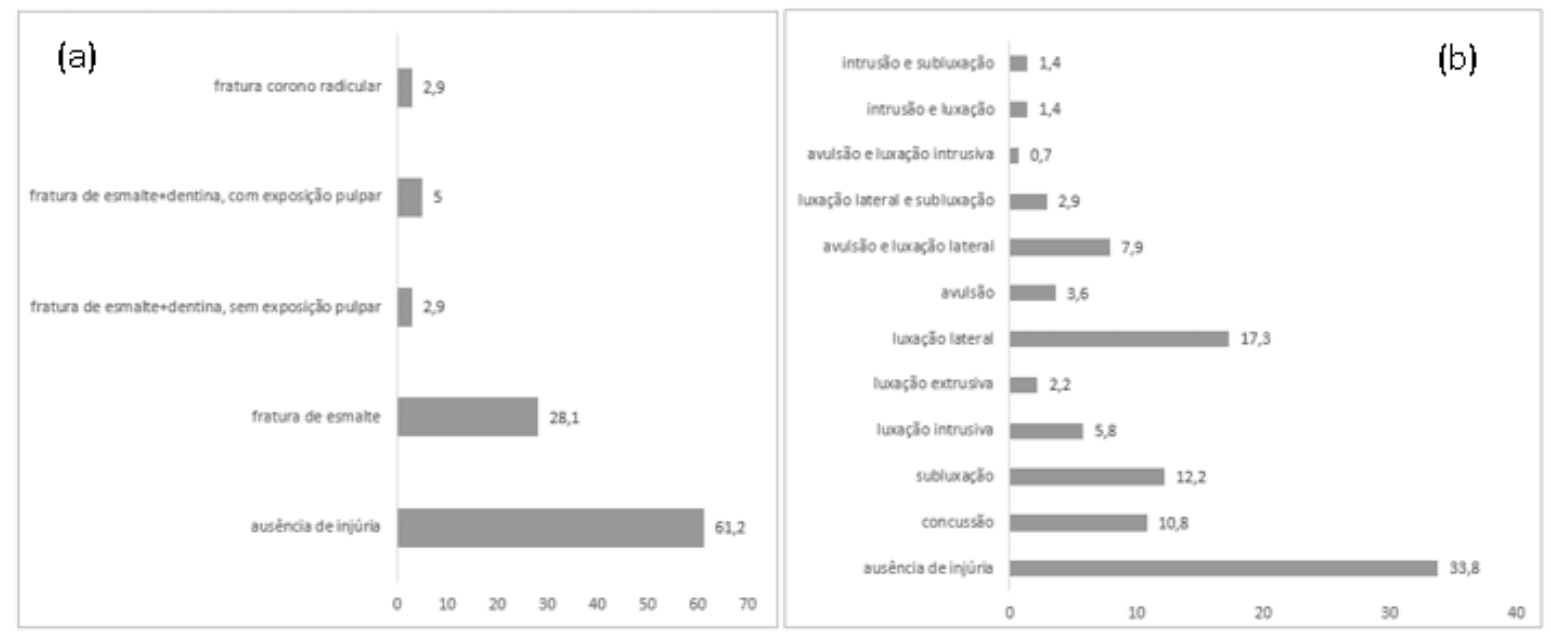

Figura 1. Prevalência de lesões traumáticas na dentição decídua, no momento do trauma (a- Injúria no dente; b- Injúria nos tecidos de suporte)

O tratamento realizado no primeiro 9,4\% entre 24 a 35 e para $19,3 \%$ este tempo foi superior atendimento, para a maioria das crianças (58,3\%) a 36 meses. Avaliando-se os dentes decíduos, ficou foi o acompanhamento, embora $14,4 \%$ necessitaram diagnosticado ausência de sinais clínicos de sequela em de exodontia, $10,1 \%$ endodontia, $8,6 \%$ tratamento restaurador e outros tratamentos $(9,3 \%)$.

$41,7 \%$, porém observou-se também ausência do dente traumatizado em $23,7 \%$, descoloração da coroa em $18 \%$

A Figura 2, demonstra os dados referente à proservação destes traumatismos, apontando que 0 tempo de acompanhamento dos pacientes foi em 57,6\% menor de 12 meses, em 13,7\% entre 12 a 23 meses, para e aparecimento de sequelas mais severas. Também foram observadas sequelas radiográficas importantes, como reabsorção radicular inflamatória (7,9\%), lesão periapical (6,5\%) calcificação pulpar (4,3\%). 


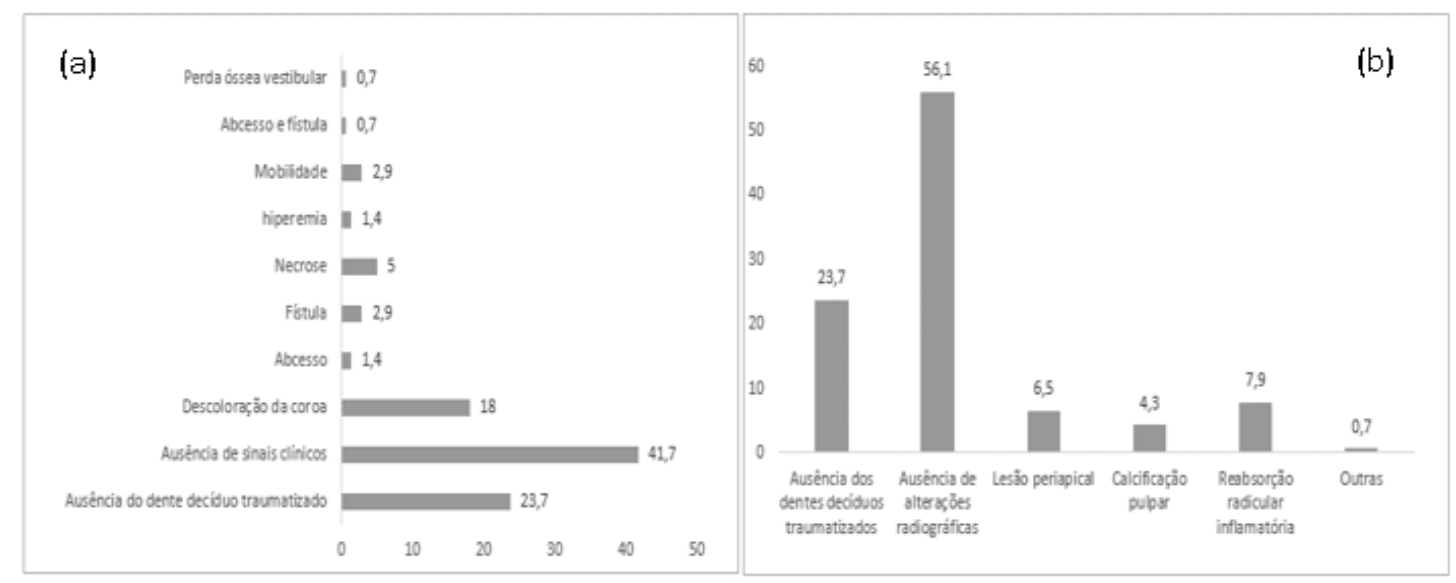

Figura 2. Prevalência de sequelas nos dentes decíduos (a- Sinais clínicos; b- Sinais radiográficos).

A Figura 3, expressa a avaliação para os dentes presença de hipoplasia de esmalte. Radiograficamente, permanentes, apontando que $13,7 \%$ dos dentes já estavam 2,2\% apresentaram atraso na erupção e 2,2\% posição irrompidos, podendo se constatar em 4 deles $(2,9 \%)$ anormal do dente.

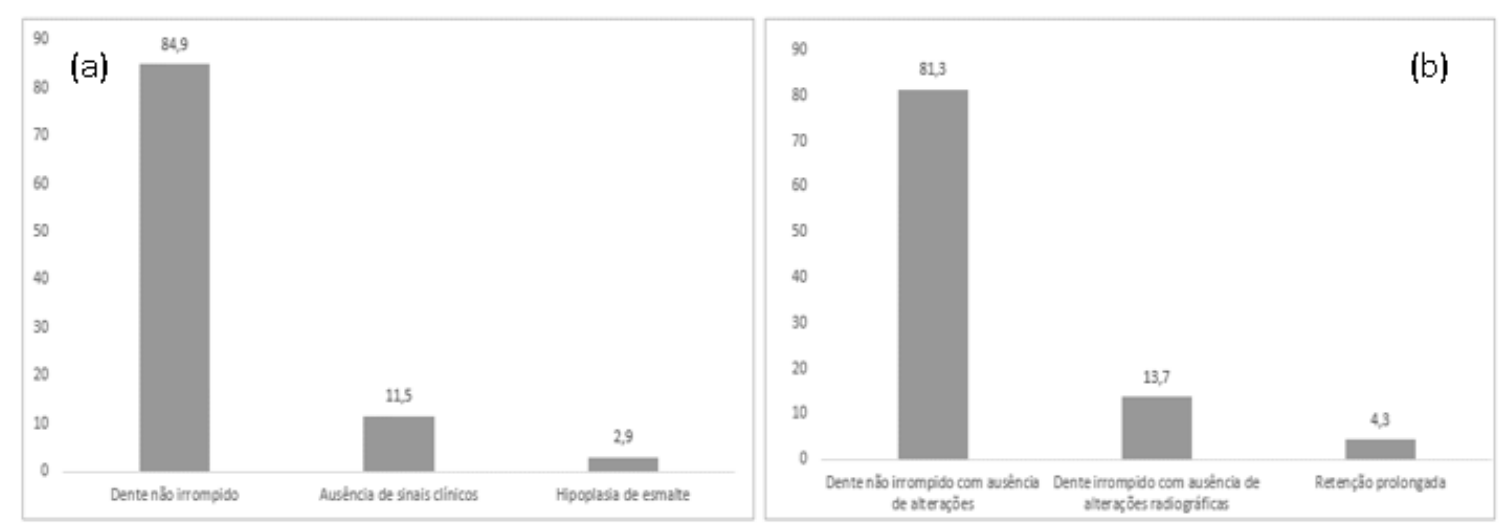

Figura 3. Prevalência de sequelas nos dentes permanentes (a- Sinais clínicos; b- Sinais radiográficos)

Por meio do Teste Qui-Quadrado, foram realizados testes associando-se as variáveis relacionadas ao trauma, discorridas até o presente momento, com o gênero da criança, não havendo associação significativa para nenhuma das variáveis estudadas $(p<0,05)$.

Já quando analisadas a associação entre as variáveis relacionadas ao trauma com a idade da criança na ocorrência do trauma, foi observada diferença estatisticamente significante entre as variáveis: injúria dentária $(p=0,02)$; tipo de injúria dentária $(p=0,01)$ e sequelas clínicas nos dentes decíduos $(p=0,02)$, como observada na tabela 1 . 
Tabela 1. Características relacionadas ao trauma dos dentes decíduos segundo a idade da ocorrência do trauma $(\mathrm{N}=139)$

\begin{tabular}{|c|c|c|c|}
\hline História do trauma & $\begin{array}{c}\leq 2 \text { anos } \\
N(\%)\end{array}$ & $\begin{array}{c}>2 \text { anos } \\
N(\%)\end{array}$ & $\begin{array}{c}\text { Valor de } \\
P\end{array}$ \\
\hline \multicolumn{3}{|l|}{ Causa } & \multirow{5}{*}{0,31} \\
\hline Colisão & $15(53,6)$ & $13(46,4)$ & \\
\hline Queda da própria altura & $47(45,2)$ & $57(54,8)$ & \\
\hline Não sabe & $1(16,7)$ & $5(83,3)$ & \\
\hline Prática de esporte & $0(0)$ & $1(100)$ & \\
\hline \multicolumn{3}{|l|}{ Número de dentes afetados } & \multirow{4}{*}{0,88} \\
\hline Apenas 1 & $30(44,8)$ & $37(55,2)$ & \\
\hline Dois dentes & $29(47,5)$ & $32(52,5)$ & \\
\hline Três ou +1 & $4(40)$ & $6(60)$ & \\
\hline \multicolumn{3}{|l|}{ Mais de um dente afetado } & \multirow{3}{*}{0,84} \\
\hline Não & $30(44,8)$ & $37(55,2)$ & \\
\hline Sim & $33(46,5)$ & $38(53,5)$ & \\
\hline \multicolumn{3}{|l|}{ História prévia de trauma } & \multirow{3}{*}{0,33} \\
\hline Não & $57(44,2)$ & $72(55,8)$ & \\
\hline Sim & $6(60)$ & $4(40)$ & \\
\hline \multicolumn{3}{|l|}{ Injúrias dentais } & \multirow{3}{*}{0,02} \\
\hline Não & $32(37,6)$ & $53(62,4)$ & \\
\hline Sim & $30(57,7)$ & $22(42,3)$ & \\
\hline \multicolumn{3}{|l|}{ Tipo de injúria dentária } & \multirow{6}{*}{0,01} \\
\hline Ausência de injúria & $32(37,6)$ & $53(62,4)$ & \\
\hline Fratura de esmalte & $21(53,8)$ & $18(46,2)$ & \\
\hline Fratura esmalte + dentina sem exposição & $1(25)$ & $3(75)$ & \\
\hline Fratura esmalte + dentina com exposição & $6(85,7)$ & $1(14,3)$ & \\
\hline Fratura coronoradicular & $3(100)$ & $0(0)$ & \\
\hline \multicolumn{3}{|l|}{ Injúrias aos tecidos de suporte } & \multirow{3}{*}{0,45} \\
\hline Não & $24(51,1)$ & $24(50)$ & \\
\hline Sim & $39(43,3)$ & $51(56,7)$ & \\
\hline \multicolumn{3}{|l|}{ Sequelas dentes decíduos - Sinais clínicos } & \multirow{4}{*}{0,02} \\
\hline Ausência dentes traumatizados & $10(30,3)$ & $23(69,7)$ & \\
\hline Ausência de sinais clínicos & $36(62,7)$ & $22(37,3)$ & \\
\hline Presença de sinais clínicos & $16(34,8)$ & $30(65,2)$ & \\
\hline \multicolumn{3}{|l|}{ Sequelas dentes decíduos - Sinais Radiográficos } & \multirow{4}{*}{0,06} \\
\hline Ausência dentes traumatizados & $10(30,3)$ & $23(69,7)$ & \\
\hline Ausência de alterações radiográficas & $42(53,8)$ & $36(46,2)$ & \\
\hline Com alterações radiográficas & $11(40,7)$ & $16(59,3)$ & \\
\hline \multicolumn{3}{|l|}{ Sequelas dentes permanentes - Sinais clínicos } & \multirow{4}{*}{0,21} \\
\hline Dente não irrompido & $57(48,3)$ & $61(51,7)$ & \\
\hline Ausência de sinais clínicos & $4(25)$ & $12(75)$ & \\
\hline Hipoplasia de esmalte & $2(50)$ & $2(50)$ & \\
\hline \multicolumn{3}{|c|}{ Sequelas dentes permanentes - Sinais Radiográficos } & \multirow{4}{*}{0,12} \\
\hline Dente não irrompido & $54(47,8)$ & $59(52,2)$ & \\
\hline Dente irrompido com ausência de Alteração & $5(26,3)$ & $14(73,7)$ & \\
\hline Presença de alteração & $4(66,7)$ & $2(33,3)$ & \\
\hline
\end{tabular}

Fonte: Dados da pesquisa 


\section{DISCUSSÃO}

O traumatismo na dentadura decídua é bastante frequente, tornando-se imprescindível que o cirurgião dentista esteja apto a atender estes casos, de modo a transmitir tranquilidade ao paciente e a seus familiares. São, por sua vez, considerados procedimentos de urgência, não só pelo envolvimento dentário, mas também pelo envolvimento emocional da criança $\mathrm{e}$ seus acompanhantes. $\mathrm{O}$ estabelecimento de um correto diagnóstico e uma estratégia terapêutica eficaz, são condutas importantes para minimizar as possíveis alterações que podem comprometer os dentes decíduos e seus sucessores permanentes em longo prazo.

O presente estudo, retrospectivo, avaliou 139 crianças, e observou uma maior prevalência de traumatismos em meninos, especialmente quando analisadas idades menores. Essa prevalência é explicada pelos fatores comportamentais, posto que a sociedade tende a estimular os meninos a serem mais competitivos (ATABEKET al., 2014). No entanto, quando analisados estatisticamente as variáveis envolvidas com o traumatismo e o gênero da crianças estudadas, este estudo não demonstrou a ocorrência de associação significativa entre as mesmas, corroborando com os achados de Coutinho, Cajazeira (2011) e Elkarmi et al. (2015), os quais afirmam não existir diferenças significativas na prevalência de lesões na dentição decídua entre ambos os gêneros, masculino e feminino, o que contrapõe os resultados de Govindarajan et al. (2012); Bücher et al. (2013); Toprak et al. (2014); Qassemet al. (2014); Atabek et al. (2014); Holan et al. (2015).

Em conformidade com os relatos encontrados em pesquisa anteriores, este estudo constatou que a faixa etária de maior prevalência na ocorrência do trauma foi entre 24 - 36 meses, sendo aos dois anos a idade mais frequentemente afetada (GÜNGÖR; PÜŞMAN; UYSAL, 2011 e ELKARMI et al., 2015). Diferentemente, para Costa et al. (2014) a faixa etária mais acometida foi entre 13 a 24 meses, enquanto que para Arikan, Sari e Sonmez (2010) e Holan, Needleman (2014) foi de 2-4 anos de idade.

Revendo a literatura relacionada com esta temática, encontra-se como unanime o apontamento por parte dos pesquisadores em relatar as quedas como o principal fator etiológico dos traumatismos na primeira infância (KRAMER et al., 2003, ALTUN et al., 2009, GOVINDARAJAN et al.,2012, TOPRAK et al., 2014). Conforme Govindarajanet al. (2012) e MendonzaMendonza et al. (2015), elas estão relacionadas com andar ou correr, visto que nos primeiros anos de vida a criança apresenta coordenação motora pouco desenvolvida. Em geral os acidentes ocorrem dentro ou próximo de casa, confirmando os dados deste estudo, onde $74,8 \%$ dos traumatismos, ocorreram devido à queda da própria altura. Elkarmiet al. (2015) constataram ser a mordida aberta um fator importante no aumento da prevalência do trauma entre as crianças, relatando no estudo uma probabilidade de ocorrência de trauma de 1,89 vezes maior quando o overjet foi maior que $3 \mathrm{~mm}$; 1,93 vezes maior nas crianças com mordida aberta anterior, e 2,56 vezes maior em crianças com cobertura labial inadequada.

Quando se analisou o número de dentes envolvidos no trauma, identificou-se que $48,2 \%$ das apresentaram trauma em um dente, $7,2 \%$ três ou mais dentes, e o restante, $43,9 \%$ em dois dentes, totalizando 218 dentes decíduos traumatizados. Arikan, Sari e Sonmez (2010) encontraram frequência dos traumatismos envolvendo dois dentes em $58,8 \%$ das crianças pesquisadas. Govindarajan et al. (2012), apontaram resultados diferentes da presente pesquisa, encontrando alta prevalência de trauma envolvendo um único dente (73,4\%). Costa et al. (2014) estudando a ocorrência de traumatismos severos em crianças pequenas, encontrou associação entre o número de dentes envolvidos e a gravidade, sendo as lesões mais graves as que envolveram três ou mais dentes.

Em geral os incisivos centrais superiores são mais acometidos por trauma (JESUS et al., 2010; GOVINDARAJAN et al., 2012; VIEGAS et al., 2014), e embora menos prevalente, neste estudo, quatro incisivos inferiores foram envolvidos em traumatismos, sendo que em três deles houve a avulsão dos elementos dentários. Em conformidade com outros estudos a maxila foi o arco mais atingido por trauma na dentição decídua (KRAMER et al., 2003; ASSUNÇÃO et al., 2009; JESUS et al., 2010; COUTINHO; CAJAZEIRA, 2011; BÜCHER et al., 2013; VIEGAS et al., 2015; BERTI et al.,2015; ELKARMI et al., 2015). Outro dado importante, diagnosticado na 
anamnese foi que $7,2 \%$ das crianças já tinham história prévia de trauma, o que segundo Malmgren et al. (2012) seria a causa para perda precoce do dente decíduos. Relatos coletados na anamnese apontaram também envolvimento dos tecidos moles $35,9 \%$, ocasionando aos bebês dificuldades para se alimentar.

Quando avaliados clinicamente os dentes decíduos, ficou constatado que $61,2 \%$ das crianças não apresentaram injúrias dentais. Foram diagnosticadas 52 crianças com alterações na coroa dentária, dentre elas se destaca as fraturas de esmalte $(28,1 \%)$, seguido da fratura de esmalte e dentina com exposição pulpar (5,0\%). Estes dados estão em consonância com os resultados obtidos por Bertiet al. (2015), comprovando no estudo que 63,4\% das sequelas avaliadas foram fraturas restritas ao esmalte. Embora sejam consideradas, fraturas não complicadas, exigindo pouco ou nenhum tratamento imediato, requerem do profissional acompanhamento clínico e radiográfico a longo prazo, quando se considera que a isquemia pulpar gerada no momento do trauma, pode levar a uma hiperemia e em alguns casos a subsequente necrose pulpar do dente decíduo (MALMGREN et al., 2012).

De acordo com os resultados encontrados neste estudo quanto às lesões dos tecidos periodontais $(n=90)$, observou-se maior frequência para a luxação lateral $(17,3 \%)$, seguido da subluxação (12,2\%), e a concussão (10,8\%). Quando o paciente apresentou mais de um dente comprometido no traumatismo, a avulsão em conjunto com a luxação, esteve presente em 13,6\%. Estes dados foram identificados por Assunção et al. (2011) e Atabek et al. (2014) que concluíram ser a subluxação a lesão mais prevalente em traumatismos na dentição decídua. Holan,Yodko e Sheinvald-Shusterman (2015) relataram que sequelas resultantes da perda precoce de incisivos decíduos podem comprometer a estética, qualidade de vida, alimentação, fonética, integridade do arco, desenvolvimento e erupção dos sucessores permanentes, além de desenvolvimento de hábitos bucais.

O tratamento realizado para maioria das crianças $(58,3 \%)$ foi o acompanhamento, embora $14,4 \%$ necessitaram de exodontia, 10,1\% endodontia, 8,6\% tratamento restaurador e outros tratamentos $(9,3 \%)$, resultados semelhantes àqueles obtidos por Gomes et al. (2010) e Toprak et al. (2014).

Na proservação destes traumatismos, o tempo de acompanhamento dos pacientes se deu em $57,6 \%$ deles com um tempo menor de 12 meses, $13,7 \%$ tempo de 12 a 23 meses, $9,4 \%$ de 24 a 35 meses e 19,3\% tempo maior de 36 meses. Dentre os 218 dentes decíduos traumatizados avaliados, em 46 crianças (33,1\%), observou-se alterações clínicas advindas do trauma. Constatou-se ser a descoloração da coroa $(25,0 \%)$ e necrose pulpar $(5,0 \%)$ a sequela clínica mais prevalente. Deste modo, acreditase que nas avaliações subsequentes destes pacientes, sequelas adicionais serão diagnosticadas, já que na grande maioria o tempo decorrido do trauma foi pequeno. Cardoso, Rocha (2010), afirmam haver associação entre a descoloração coronária com o estado da polpa, sugerindo que os dentes decíduos traumatizados com descoloração da coroa, são cinco vezes mais propensos a apresentar necrose pulpar. Qassen et al. (2014) investigaram a associação entre o nível de maturidade radicular dos dentes decíduos e as sequelas de traumatismo, verificando que houve associação significativa entre o tipo de lesão e a descoloração da coroa $(P=0,008)$, bem como entre o tipo de lesão e o desenvolvimento de calcificação pulpar $(P=0,001)$ em dentes com ápice fechado no momento do trauma. Contudo essas associações não foram encontradas para os dentes decíduos com raízes imaturas ou aqueles que se apresentaram com reabsorção radicular fisiológica.

Com respeito à avaliação radiográfica dos dentes decíduos, em 19,4\% dos dentes examinados diagnosticou-se alguma alteração radiográfica. A mais frequente foi a reabsorção radicular inflamatória (7,9\%), lesão periapical $(6,5 \%)$ e calcificação pulpar (4,3\%). Cardoso, Rocha (2010) observaram que 51 dos dentes decíduos traumatizados apresentaram alguma alteração radiográfica, e 34 alterações clínicas, destas a reabsorção radicular $(45,1 \%)$ foi a sequela radiográfica mais frequente, seguida por lesão periapical (373\%). De acordo com Güngör, Püşman e Uysal (2011) o exame radiográfico periódico deve ser realizado frequentemente, a fim de detectar precocemente as possíveis sequelas pós-trauma, já que a luxação intrusiva por exemplo, pode gerar uma distorção ou deslocamento da bainha da 
raiz epitelial de Hertwig, levando a duplicação de raiz, luxação de raiz ou cessação completa da formação da raiz do sucessor permanente. Toprak et al. (2014) consideram o acompanhamento como sendo o tratamento de escolha em dentes decíduos que sofreram trauma. Malmgren et al. (2012), elaboraram diretrizes com o objetivo de delinear uma abordagem para o cuidado imediato ou urgente, para uma melhor conduta frente a lesões em dentes decíduos, como método de prevenção de sequelas para os dentes permanentes.

De acordo com os resultados obtidos em 4,4\% dos dentes observou-se poucas alterações clínicas e radiográficas nos sucessores permanentes. As alterações clínicas identificadas foram a hipoplasia de esmalte (2,9\%) e radiograficamente a retenção prolongada $(4,4 \%)$, mas com citado anteriormente, o tempo decorrido do trauma foi pequeno, assim como o número de dentes permanentes irrompidos. Carvalho, Jacomo e Campos (2010); Amorin et al. (2011) estabeleceram com seus estudos que no que diz respeito aos sucessores permanentes, que as sequelas mais encontradas foram a alteração de cor e hipoplasia de esmalte. Skaare et al. (2013) observaram que $41,7 \%$ dos sucessores permanentes apresentaram sequelas de desenvolvimento, dentre elas o mais prevalente foi descoloração do esmalte e hipoplasia de esmalte. Altun et al. (2009), concluíram não haver nenhuma correlação significativa entre a idade no momento da intrusão, e a frequência de distúrbios no desenvolvimento, embora reforcem que o germe do incisivo central superior permanente se forme por volta na $20^{\mathrm{a}}$ semana de gestação, porém a calcificação não inicia-se antes de 3-4 meses de idade, e quando há um trauma pode ocorrer uma pausa no desenvolvimento da coroa, com destruição dos ameloblastos do epitélio do esmalte ativo podendo resultar em hipoplasia do esmalte.

Güngör, Püşman e Uysal (2011) estudaram a influência do trauma dos dentes decíduos, para 0 sucessor permanente, e concluíram que esta pode estar relacionada com diversos fatores, como a relação do espaço entre os dentes envolvidos, o grau de reabsorção da raiz do decíduo no momento da lesão, a direção da força traumática, o tipo de lesão para o incisivo decíduo, fase de desenvolvimento do germe do dente permanente, e a idade da criança no momento da lesão; sendo a fase de maior risco até os 2 anos de idade, visto que, o germe dos dentes permanentes são sensíveis nas fases iniciais do seu desenvolvimento, que ocorre no período entre o quarto mês e quatro anos. Neste momento crítico, lesões ou alterações inflamatórias podem interferir nos diferentes níveis da odontogênese, tais como a diferenciação morfológica, organização, mineralização, ou a maturação final pré eruptiva.

Diante dos dados coletados no presente estudo e da consulta na literatura cientifica, fica clara a importância do retorno do paciente nas consultas de avaliação, como um método de prevenção de sequelas pós-trauma, sobretudo em crianças pequenas, onde o risco é mais presente. Portanto, cabe ao cirurgião dentista, no momento do primeiro atendimento, orientar os pais e acompanhantes acerca da importância das consultas de avaliação, como um método de prevenção de sequelas para os dentes decíduos e germes dos dentes permanentes, lembrando-os sempre que a maior resiliência do osso ao redor dos dentes decíduos e a pouca idade da criança são fatores importantes no desenvolvimento de alterações.

Contudo, esta pesquisa apresentou limitações, dentre elas está o tamanho pequeno da amostra e o pouco tempo decorrido pós-traumatismo, comprometendo a análise mais precisa dos dados estatísticos e a associação entre as variáveis estudadas. Portanto, torna-se necessário, em trabalhos futuros, que a amostra seja expandida e que o tempo para avaliação seja mantido até que o dente permanente faça sua irrupção na cavidade bucal.

\section{CONCLUSÃO}

Os traumatismos dentários são considerados um grave problema de saúde pública, especialmente em crianças pequenas; devendo os pais e acompanhantes serem informados sobre a prevenção e cuidados frente as lesões dentárias traumáticas, bem como a grande importância do acompanhamento dos dentes traumatizados até a irrupção do dente permanente. 


\section{REFERÊNCIAS}

ALTUN, C.; CEHRELI, Z. C.; GÜVEN, G.; ACIKEL, C. Traumatic intrusion of primary teeth and its effects on the permanent sucessors: a clinical follow-up study. Oral Surg Oral Med Oral Pathol Oral Radiol, v.107, n.4, p.493-498, 2009.

AMORIM, L. F. G.; ESTRELA, C.; COSTA, L. R. R. S. Effects of traumatic dental injuries to primary teeth on permanent teeth - a clinical follow-up study. Dent Traumatol, v.27, n.2, p.117-121, 2011.

ANDREASEN, J.O.; ANDREASEN, F.M.; ANDERSSON, L. Textbook and color atlas of traumatic injuries to the teeth. $4^{\text {th }}$ ed. Oxford: BlackwellMunksgaard; 2007.

ARIKAN, V.; SARI, S.; SONMEZ, H. The Prevalence and Treatment Outcomes of Primary Tooth Injuries. European J Gen Dent, v.4, n.4, p.447-453, 2010.

ASSUNÇÃO, L.R.S.; FERELLE, A.; IWAKURA, M.L.; NASCIMENTO, L.S.; CUNHA, R.F. Luxation injuries in primary teeth: a retrospective study in children assisted at an emergency service. Braz Oral Res, v.25, n. 2, p. 150-156, 2011.

ASSUNÇÃO, L. R. S.; FERELLE, A.; IWAKURA, M.L.H.; CUNHA, R.F. Effects on permanent teeth after luxation injuries to the primary predecessors: a study in children assisted at an emergency service. Dent Traumatol, v.25, n.2, p.165-170, 2009.

ATABEK, D.; ALAÇAM, A.; AYDINTUG, I.; KONAKOGLU, G. A retrospective study of traumatic dental injuries. Dent Traumatol, v.30, n.2, p.154-161, 2014.

BERTI, G. O.; HESSE,D.; BONIFÁCIO,C. C.; RAGGIO, D. P.; BÖNECKER, M. J. S.; Epidemiological study of traumatic dental injuries in 5- to 6- year-old Brazilian children. Braz Oral Res, v.29, n.1, p.1-6, 2015.

BÜCHER, K.; NEUMANN, C.; HICKEL, R; KÜHNISCH, J. Traumatic dental injuries at a German University Clinic 2004-2008. Dent Traumatol, v.29, n.2, p. 127-133, 2013.

CARDOSO, M.; ROCHA, M.J.C. Association of crown discoloration and pulp status in traumatized primary teeth. Dent Traumatol, v.26, n.5, p. 413-416, 2010.
CARVALHO, V.; JACOMO, D. R.; CAMPOS, V. Frequency of intrusive luxation in deciduous teeth and its effects. Dent Traumatol, v.26, n.4, p.304-307, 2010.

COSTA, V.P.P.; BERTOLDI, A.D.; BALDISSERA, E.Z.; GOETTEMS, M.L.; CORREA, M.B.; TORRIANI, D.D. Traumatic dental injuries in primary teeth: severity and related factors observed at a specialist treatment centre in Brazil. Eur Arch Paediatr Dent, v.15, n.2, p. 83-88, 2014.

COUTINHO, T.C.; CAJAZEIRA, M. R. Retrospective study on the occurrence of primary incisor trauma in preschool children of a low-income area in Brazil. EEur Arch Paediatr Dent, v. 12, n.3, p. 159-62, 2011.

ELKARMI, R.F.; HAMDAN, M.A.; RAJAB, L.D.; ABUGHAZALEH, S.B.; SONBOL, H.N. Prevalence of traumatic dental injuries and associated factors among preschool children in Amman, Jordan. Dent Traumatol, v. 31, n. 6, p. 487-92, 2015.

GOMES, A.C.; MESSIAS, L.P.; DELBEM, A.C., CUNHA, R.F. Developmental disturbance of an unerupted permanent incisor due to trauma to its predecessor. J Can Dent Assoc, p. 57-76, 2010.

GOVINDARAJAN, M.; REDDY, V.N.; RAMALINGAM, K.; DURAI, K.S.; RAO, P.A.; PRABHU,A. Prevalence of traumatic dental injuries to the anterior teeth among three to thirteen-year-old school children of Tamilnadu. Contemp Clin Den, v.3, n.2, p. 164-7, 2012.

GÜNGÖR, H.C.; PÜŞMAN, E.; UYSAL, S. Eruption delay and sequelae in permanent incisors following intrusive luxation in primary dentition: a case report. Dent Traumatol, v.27, n.2, p.156-158, 2011.

HOLAN, G.; YODKO, E.; SHEINVALD-SHUSTERMAN, K.; The association between traumatic dental injurie and atypical external root resorption in maxillary primary incisors. Dent Traumatol, v.31, n.1, p.35-41, 2015.

HOLAN, G.; NEEDLEMAN, H .L. Premature loss of primary anterior teeth due to trauma - potential short- and longterm sequelae. Dent Traumatol, v.30, n.2, p.100-106, 2014.

JÁCOMO, D.R.E.S.; CAMPOS, V. Prevalence of sequelae in the permanent anterior teeth after trauma in their 
predecessors: a longitudinal study of 8 years. Dent Traumatol, v.25, n.3, p.300-304, 2009.

JESUS, M.A.; ANTUNES, L.A.; RISSO P.A.; FREIRE, M.V.; MAIA, L.C. Epidemiologic survey of traumatic dental injuries in children seen at the Federal University of Rio de Janeiro, Brazil. Braz Oral Res, v. 24, n.1, p. 89-94, 2010.

KRAMER, P. F.; ZEMBRUSKI, C.; FERREIRA, S. H; FELDENS, C. A. Traumatic dental injuries in Brazilian preschool children. Dent Traumatol, v. 19, n. 6, p. 299 303, 2003.

MALMREN, B.; ANDREASEN, J.O.; FLORES, M.T.; ROBERTSON, A.; DIANGELLIS, A. J.et al. International Association of Dental Traumatology guidelines for the management of traumatic dental injuries: 3. Injuries in the primary dentition. Dent Traumatol, v. 28, n.3, p. 174-182, 2012.

MENDONZA-MENDONZA， A.; LINARES-IGLESIAS， A.; VICO-YAÑEZ, R.M.; LUBRUZZI-ABALLOS, C. Prevalence and complications of trauma to the primary dentition in a subpopulation of Spanish children in southern Europe. Dent Traumatol, v.31, n.2, p.144-149, 2015.

OZDEMIR, Y; AKIN, A; EDEN, E. Management of multiple sequelaes in permanent dentition: 3 years follow-up. Dent Traumatol, v. 27, n.1, p.67-70, 2011.

QASSEM, A.; GOETTEMS, M.; TORRIANI, D.D.; PAPPEN, F.G. Radicular maturity level of primary teeth and its association with trauma sequelae. Dent Traumatol, v.30, n.3, p.227-231, 2014.

SKAARE, A. B; A.A.S.; A. M.; WANG, N. J. Enamel defects in permanent incisors after trauma to primary predecessors: inter-observer agreement based on photographs. Dent Traumatol, v. 29, n. 2, p. 79-83, 2013.

TOPRAK, M.E.; TUNA, E.B.; SEYMEN, F.; GENÇAY, K. Traumatic dental injuries in Turkish children, Istanbul. Dent Traumatol, v.30, n.4, p.280-284, 2014.

VIEGAS, C. M.; PAIVA, S. M.; CARVALHO, A. C.; SCARPELLI, A. C.; FERREIRA, F.M.; PORDEUS, I. A. Influence of traumatic dental injury on quality of life of Brazilian preschool children and their families. Dent Traumatol, v. 30, n. 5, p. 338-347, 2014.

ZHANG, Y.; ZHU, Y.; SU, W.; ZHOU, Z.; JIN, Y.; WANG,
X. A retrospective study of pediatric traumatic dental injuries in Xi'an, China. Dent Traumatol, v.30, n.3, p. 211-215, 2014.

Recebido em: 06 de maio de 2016 Aceito em: 07 de setembro de 2016 